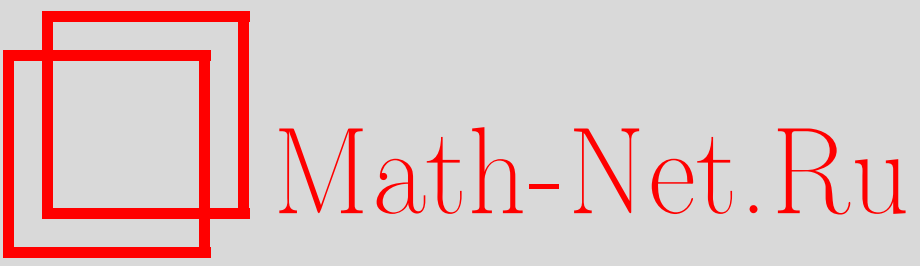

С. В. Петрушкин, В. В. Самарцев, Метод исключения бозонных операторов в проблеме лазерного охлаждения твердых тел, ТМФ, 2001, том 126, номер 1, 164-174

DOI: https://doi.org/10.4213/tmf422

Использование Общероссийского математического портала Math-Net.Ru подразумевает, что вы прочитали и согласны с пользовательским соглашением

http://www. mathnet.ru/rus/agreement

Параметры загрузки:

IP : 3.95 .254 .165

26 апреля 2023 г., 14:49:21

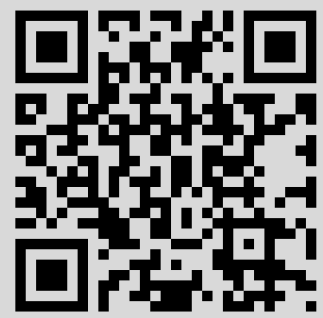


ТЕОРЕТИЧЕСКАЯ

И МАТЕМАТИЧЕСКАЯ

ФИЗИКА

Том 126, № 1

январь, 2001

(C) 2001 г.

С.В. Петрушкин ${ }^{*}$, В.В. Самарцев ${ }^{\dagger}$

\section{МЕТОД ИСКЛЮЧЕНИЯ БОЗОННЫХ ОПЕРАТОРОВ В ПРОБЛЕМЕ ЛАЗЕРНОГО ОХЛАЖДЕНИЯ ТВЕРДЫХ ТЕЛ}

С помощью метода исключения бозонных переменных Боголюбова исследуется проблема лазерного охлаждения твердотельных образцов. Выведено точное кинетическое уравнение для квантового описания процесса лазерного охлаждения стекол и кристаллов, легированных редкоземельными ионами, и найдено его стационарное решение. Оценена температура лазерно-охлажденного стекла, легированного трехвалентным иттербием.

\section{1. ВВЕДЕНИЕ}

Проблема лазерного охлаждения твердотельных образцов является одной из наиболее актуальных и практически значимых в лазерной физике [1]. Хорошо известны успехи в лазерном охлаждении газов [2], [3], подтвержденные присуждением в 1997 году нобелевской премии С. Чу, К. Коен-Таннуджи и В. Филлипсу. Достижение таких же успехов в лазерном охлаждении твердых тел позволило бы создать миниатюрные лазерные рефрижераторы, функционируюшие без использования криогенных жидкостей. В настояшее время стоит задача проведения таких экспериментов на твердотельных образцах. В основе этих экспериментов лежит антистоксов режим излучения поглощенной энергии, когда испускаемый средой квант света больше кванта поглощенного света на величину энергии фонона. На рис. 1 приведена схема антистоксова режима лазерного охлаждения твердых тел, где $\hbar \Omega$ - квант фонона, $\hbar \omega-$ квант фотона накачки, $\hbar(\omega+\Omega)$ - квант фотона флуоресценции. Энергия фонона уносится из образца в результате флуоресценции.

На эффективность антистоксова режима охлаждения указал еше в 1929 году Прингсхейм [4]. Позднее Кастлер [5] обратил внимание на то, что в качестве рабочих частиц, реализующих режим лазерного охлаждения, следует использовать редкоземельные ионы благодаря их высокой квантовой эффективности. Именно редкоземельные ионы ионы трехвалентного иттербия $\left(\mathrm{Yb}^{3+}\right)$, находящиеся в тяжелометаллическом флюридном стекле (ZBLANP), были использованы Эпстейном с коллегами из Лос-Аламосской

\footnotetext{
*Казанский физико-технический институт КНЦ РАН, Казань, Россия. E-mail: serpens@kfti.knc.ru

${ }^{\dagger}$ Казанский государственный университет, Казань, Россия. E-mail: samartsev@dionis.kfti.knc.ru
} 


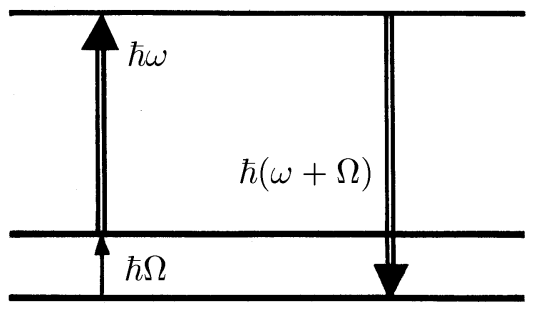

Рис. 1

национальной лаборатории (США) в 1995 году для постановки первого твердотельного эксперимента по лазерному охлаждению [6]. Структура рабочих уровней этого редкоземельного иона приведена на рис. 2 [7].

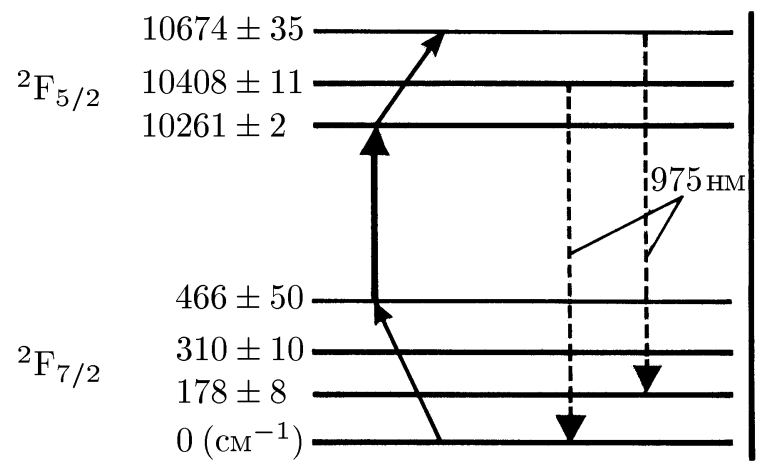

Рис. 2

Наивысшее достижение в лазерном охлаждении $\mathrm{Yb}^{3+}$ : ZBLANP составило $21^{\circ}$ ниже комнатной температуры [8]. В 1995 году был зарегистрирован патент США на имя Эпстейна, Эдвардса, Бухвальда и Госнелла “Флуоресцентный рефрижератор” [9].

С 1996 года начались наши теоретические разработки проблемы лазерного охлаждения твердых тел [10]-[15] на основе метода неравновесного статистического оператора. Результаты этих разработок суммированы нами в книге [1]. В этих работах был сделан существенный шаг в понимании процессов, протекающих в указанных экспериментах. В данной работе мы применим другой математический подход к решению проблемы, основанный на методе исключения полевых переменных, и приведем конечный результат к виду, который позволил сделать необходимые оценки температуры охлаждения и провести сравнение с экспериментом. В отличие от использованного ранее метода построения уравнений для динамических переменных системы, указанный подход, как бы вытекая из самой сути явления, обладает рядом преимуществ для поставленной задачи, и прежде всего он дает ясную физическую картину происходящих процессов.

Как известно, динамическая проблема в квантовой механике не может быть сформулирована без некоторого произвольного выбора той части системы, которая подлежит рассмотрению. Полный гамильтониан системы должен быть разбит на две составляющие. Одна из них описывает те части физической системы, переходы в которых явля- 
ются предметом рассмотрения, тогда как другая описывает взаимодействие этих частей. Часто используемое так называемое приближение заданных внешних сил [16], когда электромагнитное поле можно считать заданной функцией и вместо совокупности описывающих его величин подставлять их средние значения, обретает здесь точный характер и позволяет самосогласованным образом учесть влияние поля, явно исключив полевые операторы из уравнений для величин атомной подсистемы. Таким образом, в данном подходе вывод уравнений необходимо делать для меньшего числа динамических переменных и вся процедура сводится к вычислению коммутаторов.

\section{2. ВЫВОД КИНЕТИЧЕСКИХ УРАВНЕНИЙ}

Метод иерархий кинетических уравнений, развитый Боголюбовым и Боголюбовым (мл) [17]-[19], являющийся весьма общим при описании динамических процессов в “малой” подсистеме, приводимой в контакт с термостатом, и нашедший широкое применение в теории сверхизлучения [20], [21], может быть использован для описания широкого круга явлений в конденсированных средах.

Рассмотрим ансамбль из $N$ излучателей с частотами перехода $\Omega_{j}$ (индекс $j$ нумерует излучатели), находяшихся в рабочем объеме $V_{\mathrm{c}}=\prod_{i} L_{i}, i=x, y, z$ (например, для системы излучателей, расположенных в узлах простой кубической решетки, $V_{\mathrm{c}}=N a^{3}$, где $a$ - постоянная решетки) в условиях, когда электромагнитное поле квантуется в объеме $V \gg V_{\mathrm{c}}$.

Задан гамильтониан системы

$$
\begin{gathered}
H=H_{\mathrm{M}}+H_{\mathrm{F}}+H_{\mathrm{MF}}, \\
H_{\mathrm{F}}=\sum_{k} \hbar \omega_{k} a_{k}^{+} a_{k}, \quad H_{\mathrm{MF}}=\sum_{k}\left(M_{k}^{+} a_{k}^{+}+M_{k} a_{k}\right),
\end{gathered}
$$

где величины $M_{k}^{+}, M_{k}$ зависят только от переменных атомной подсистемы, $a_{k}^{+}\left(a_{k}\right)-$ операторы рождения (уничтожения) фотонов моды $k$ и частоты $\omega_{k}$.

Состояние общей системы (вешество+поле) задается матрищей плотности $\rho_{\mathrm{MF}}(t)$. Начальное состояние поля полагаем равновесным, а моментом начала взаимодействия будем считать $t_{0}$. Тогда

$$
\rho_{\mathrm{MF}}\left(t_{0}\right)=\rho_{\mathrm{M}}\left(t_{0}\right) \otimes \rho_{\mathrm{F}}\left(t_{0}\right) \quad \text { и } \quad \rho_{\mathrm{F}} \equiv \frac{e^{-\beta H_{\mathrm{F}}}}{\operatorname{Tr}_{\mathrm{F}}\left(e^{-\beta H_{\mathrm{F}}}\right)} .
$$

При рассмотрении динамических процессов в квантово-статистических системах отправным пунктом является уравнение Неймана

$$
i \hbar \frac{\partial \rho_{\mathrm{MF}}}{\partial t}=H \rho_{\mathrm{MF}}-\rho_{\mathrm{MF}} H,
$$

где $H$ - оператор Гамильтона, определяющий полную энергию системы. В интересующем нас случае система представляет собой совокупность некоторого числа излучателей, взаимодействуюших с электромагнитным полем, играющим роль термостата.

Для описания процесса с участием произвольного числа фотонов $s$ необходимо в слагаемом $H_{\mathrm{MF}}$ гамильтониана (1) произвести замену [22]-[25]

$$
a^{+} \rightarrow \frac{\hbar}{N^{\frac{s}{2}}} \prod_{i=1}^{s} a_{i}^{+}, \quad a \rightarrow \frac{\hbar}{N^{\frac{s}{2}}} \prod_{i=1}^{s} a_{i}
$$


внеся соответствуюшие поправки в константу взаимодействия.

Рассмотрим случай, когда $s=2$ и

$$
H=H_{\mathrm{M}}+H_{\mathrm{F}}+\sum_{k}\left(M_{k}^{+} a_{k}^{+} b^{+}+M_{k} a_{k} b\right) .
$$

Здесь операторы $b^{+}, b$ описывают моду электромагнитного поля, выделенную в силу каких-либо физических причин. Полезно заметить, что мода $b^{+}, b$ может быть не только электромагнитной природы. В задаче лазерного охлаждения она описывает фонон. В этой связи будем теперь считать, что по-прежнему взаимодействие задано гамильтонианом $H_{\mathrm{MF}}$ из (1), но с новыми “эффективными” операторами атомной подсистемы

$$
M_{k} b \rightarrow \widetilde{M}_{k} \equiv M_{k}, \quad M_{k}^{+} b^{+} \rightarrow \widetilde{M}_{k}^{+} \equiv M_{k}^{+}
$$

Пусть $o_{\mathrm{M}}(t)$ - оператор, который действует на собственные функции гамильтониана (1) только как на функции переменных, относящихся к М-системе. Вводя представление Гейзенберга для произвольной динамической величины $O_{\mathrm{MF}}$, заданной в представлении Шредингера,

$$
O_{\mathrm{MF}}(t)=U^{-1}\left(t, t_{0}\right) O_{\mathrm{MF}} U\left(t, t_{0}\right),
$$

где унитарньй оператор $U\left(t, t_{0}\right)$ удовлетворяет уравнению

$$
i \hbar \frac{\partial}{\partial t} U\left(t, t_{0}\right)=H U\left(t, t_{0}\right), \quad U\left(t_{0}, t_{0}\right)=1,
$$

запишем уравнение движения для $o_{\mathrm{M}}(t)$

$$
i \hbar \frac{\partial}{\partial t} o_{\mathrm{M}}(t)=\left[o_{\mathrm{M}}(t), H\right]
$$

Дополнительный вклад в эволюцию операторов поля в данной моде (оператора $a_{k}^{+}(t)$ или оператора $\left.a_{k}(t)\right)$ при включении взаимодействия аддитивно обусловлен лишь функциями динамических величин, относяшихся к атомной подсистеме. Действительно,

$$
\begin{aligned}
& i \hbar \frac{\partial}{\partial t} a_{k}(t)=\left[a_{k}(t), H_{\mathrm{F}}+H_{\mathrm{MF}}\right]=\hbar \omega_{k} a_{k}(t)+\sum_{k^{\prime}} M_{k^{\prime}}^{+}(t) \delta_{k^{\prime} k}, \\
& i \hbar \frac{\partial}{\partial t} a_{k}^{+}(t)=\left[a_{k}^{+}(t), H_{\mathrm{F}}+H_{\mathrm{MF}}\right]=-\hbar \omega_{k} a_{k}^{+}(t)-\sum_{k^{\prime}} M_{k^{\prime}}(t) \delta_{k^{\prime} k} .
\end{aligned}
$$

Решая (6) и (7), получаем

$$
\begin{aligned}
& a_{k}(t)=\widetilde{a_{k}}(t)+\frac{1}{i \hbar} \int_{t_{0}}^{t} d \tau M_{k}^{+}(\tau) e^{-i \omega_{k}(t-\tau)}, \\
& a_{k}^{+}(t)=\widetilde{a_{k}^{+}}(t)-\frac{1}{i \hbar} \int_{t_{0}}^{t} d \tau M_{k}(\tau) e^{i \omega_{k}(t-\tau)},
\end{aligned}
$$

где $\widetilde{a_{k}}(t)$ и $\widetilde{a_{k}^{+}}(t)$ - решения, отвечающие случаю свободного электромагнитного поля. 
Данное обстоятельство оказывается весьма полезным при составлении уравнений для средних значений операторов типа (5), вычисляемых по матрице плотности, заданной выражениями (2),

$$
i \hbar \frac{\partial}{\partial t}\left\langle o_{\mathrm{M}}(t)\right\rangle=\left\langle\left[o_{\mathrm{M}}(t), H\right]\right\rangle .
$$

Выпишем явно это уравнение, подставив гамильтониан (1) и имея в виду зависимости (8) и $(9)$ :

$$
i \hbar\left(\frac{\partial}{\partial t}\left\langle o_{\mathrm{M}}(t)\right\rangle+\frac{1}{i \hbar}\left\langle\left[H_{\mathrm{M}}, o_{\mathrm{M}}(t)\right]\right\rangle\right)=\left\langle\left[o_{\mathrm{M}}(t), H_{\mathrm{MF}}\right]\right\rangle .
$$

Тождественными преобразованиями приведем коммутатор в правой части к виду

$$
\left[o_{\mathrm{M}}(t), H_{\mathrm{MF}}\right]=\sum_{k}\left(\left[o_{\mathrm{M}}(t), M_{k}\right] a_{k}+\left[o_{\mathrm{M}}(t), M_{k}^{+}\right] a_{k}^{+}\right) .
$$

Удобство представления коммутатора в такой форме видно из нижеследуюшего.

Рассмотрим некоммутируюшие операторы $x$ и $y$, причем $\left[x, x^{+}\right]=1$. Тогда для обобщенного параметра $\lambda[26]$ имеем

$$
e^{\lambda x^{+} x} x e^{-\lambda x^{+} x}=e^{-\lambda} x,
$$

и для всякого распределения, допускаюшего разложение $\rho=\rho_{(\ldots)} e^{-\lambda x^{+} x},-$

$$
\operatorname{Tr}\{\rho x y\}=\operatorname{Tr}\left\{\rho y \rho x \rho^{-1}\right\}=e^{\lambda} \operatorname{Tr}\{\rho y x\} .
$$

Таким образом, среднее от произведения операторов $x$ и $y$ связано с их коммутатором, что и позволяет нам исключить полевые операторы из правой части (12). Справедлива следующая лемма [18].

Лемма 1. Если $O(t) \equiv O_{\mathrm{MF}}(t)$, әде индексы $\mathrm{F}, \mathrm{M}$ указывают на квантовые числа полевой и атомной систем, $a_{k}, a_{k}^{+}$- операторь бозонного поля, то имеют место следующие равенства для средних:

а) $\left\langle a_{k} O(t)\right\rangle=\left(1+N_{k}\right)\left\langle\left[a_{k}, O(t)\right]\right\rangle$,

б) $\left\langle a_{k}^{+} O(t)\right\rangle=-N_{k}\left\langle\left[a_{k}^{+}, O(t)\right]\right\rangle$, где число $N_{k}$ таково, что

\section{$\beta$ - обратная температура.}

$$
\frac{1+N_{k}}{N_{k}}=e^{\beta \hbar \omega_{k}}
$$

Выбирая в качестве $O$ коммутаторы $\left[o_{\mathrm{M}}(t), M_{k}(t)\right],\left[o_{\mathrm{M}}(t), M_{k}^{+}(t)\right]$ из формулы $(12)$, получаем окончательное искомое кинетическое уравнение (11) для системы из $N$ многоуровневых излучателей с учетом двухквантового характера процесса взаимодействия:

$$
\begin{aligned}
\frac{\partial}{\partial t}\left\langle o_{\mathrm{M}}(t)\right\rangle & +\frac{1}{i \hbar}\left\langle\left[H_{\mathrm{M}}+\hbar \omega_{b} b^{+} b, o_{\mathrm{M}}(t)\right]\right\rangle= \\
= & \sum_{k}\left\{N_{k}\left\langle\frac{1}{\hbar^{2}} \int_{t_{0}}^{t} d \tau e^{-i \omega_{k}(t-\tau)} M_{k}^{+}(\tau) b^{+}(\tau)\left[o_{\mathrm{M}}(t), M_{k}(t) b(t)\right]\right\rangle+\right. \\
& \left.+\left(1+N_{k}\right)\left\langle\frac{1}{\hbar^{2}} \int_{t_{0}}^{t} d \tau e^{-i \omega_{k}(t-\tau)}\left[M_{k}(t) b(t), o_{\mathrm{M}}(t)\right] M_{k}^{+}(\tau) b^{+}(\tau)\right\rangle\right\}+ \\
& +\sum_{k}\left\{N_{k}\left\langle\frac{1}{\hbar^{2}} \int_{t_{0}}^{t} d \tau e^{i \omega_{k}(t-\tau)}\left[M_{k}^{+}(t) b^{+}(t), o_{\mathrm{M}}(t)\right] M_{k}(\tau) b(\tau)\right\rangle+\right. \\
& \left.+\left(1+N_{k}\right)\left\langle\frac{1}{\hbar^{2}} \int_{t_{0}}^{t} d \tau e^{i \omega_{k}(t-\tau)} M_{k}(\tau) b(\tau)\left[o_{\mathrm{M}}(t), M_{k}^{+}(t) b^{+}(t)\right]\right\rangle\right\}
\end{aligned}
$$


где $\omega_{b}$ - частота квантов поля, описываемого операторами $b^{+}$и $b$, и, как отмечалось, $o_{\mathrm{M}}(t)$ - оператор, действующий на переменные М-системы, описываемой гамильтониаHOM

$$
\widetilde{H}_{\mathrm{M}}=H_{\mathrm{M}}+\hbar \omega_{b} b^{+} b .
$$

Полученная иерархия (13) описывает временную зависимость средних значений динамических переменных М-системы (излучателей). Однако на ее основе могут быть получены и характеристики излучения, если воспользоваться выражениями для интегралов движения гамильтониана (1).

\section{3. ЛАЗЕРНОЕ ОХЛАЖДЕНИЕ ТВЕРДЫХ ТЕЛ}

Рассмотрим лазерное охлаждение примесных частищ в кристалле, подсистема которых сама может служить охладителем для образца. Примесные молекулы испытывают колебательные либрации относительно своих равновесных положений в кристалле. В силу того что направления дипольного момента перехода жестко связаны с осями симметрии молекулы, эти либрации модулируют постоянную взаимодействия молекулы с электромагнитным полем, что приводит к так называемым непрямым переходам, когда вместе с фотонами поглошается или испускается фонон.

Гамильтониан анизотропных молекул в кристалле может быть записан как

$$
H=H_{\mathrm{a}}+H_{\mathrm{f}}+H_{\mathrm{v}}+H_{\mathrm{af}}+H_{\mathrm{af}}^{\mathrm{v}} .
$$

Здесь

$$
H_{\mathrm{af}}=\sum_{j k}\left(g_{k} e^{i \mathbf{k r}_{j}} S_{j}^{+} a_{k}+g_{k}^{*} e^{-i \mathbf{k r}_{j}} S_{j}^{-} a_{k}^{+}\right)
$$

- гамильтониан взаимодействия между молекулами и электромагнитным полем в приближении врашаюшейся волны, $g_{k}$ - константа взаимодействия, $\mathbf{k}$ - волновой вектор фотона моды $k, \mathbf{r}_{j}$ - радиус-вектор $j$-й молекулы, $S_{j}^{ \pm}$- оператор повышения и понижения проекции эффективного спина;

$$
H_{\mathrm{af}}^{\mathrm{v}}=\sum_{j m k}\left(h_{m k} e^{i \mathbf{k r}_{j}} S_{j}^{+}\left(B_{j m}+B_{j m}^{+}\right) a_{k}+h_{m k}^{*} e^{-i \mathbf{k r}_{j}} S_{j}^{-}\left(B_{j m}+B_{j m}^{+}\right) a_{k}^{+}\right)
$$

- гамильтониан взаимодействия между молекулами и электромагнитным полем с участием фононов, $h_{m k}-$ постоянная взаимодействия.

Кратко остановимся на физических механизмах, приводящих к охлаждению конденсированных систем лазерным излучением. Закон сохранения энергии для процесса, описываемого гамильтонианом (14), может быть записан как

$$
\begin{aligned}
\sum_{k} \hbar \omega_{k} \frac{d\left\langle n_{k}\right\rangle}{d t}+\sum_{m} \hbar \Omega_{m} \frac{d\left\langle n_{m}\right\rangle}{d t}+\hbar \omega_{0} \frac{d\left\langle S^{z}\right\rangle}{d t}= \\
=\sum_{k} \omega_{k} \frac{\tilde{n}_{k}-\left\langle n_{k}\right\rangle}{\tau_{k}}+\sum_{m} \hbar \Omega_{m} \frac{\tilde{n}_{m}-\left\langle n_{m}\right\rangle}{\tau_{m}},
\end{aligned}
$$

где $\tilde{n}_{k}$ - заданное внешним источником среднее число фотонов моды $k ; \tilde{n}_{m}$ - определяемое взаимодействием с термостатом среднее число фононов моды $m$. 
В стационарном режиме, когда все производные равны нулю, из (17) получаем

$$
\sum_{k} \hbar \omega_{k} \frac{\tilde{n}_{k}}{\tau_{k}}-\sum_{k} \hbar \omega_{k} \frac{\left\langle n_{k}\right\rangle}{\tau_{k}}+\sum_{m} \hbar \Omega_{m} \frac{\tilde{n}_{m}-\left\langle n_{m}\right\rangle}{\tau_{m}}=0 .
$$

Пусть возбуждение имеет место лиш на определенной частоте $\omega_{e}$, а излучение происходит на этой же частоте и на более высокой частоте

$$
\omega_{f}=\omega_{e}+\Omega_{m} .
$$

Тогда уравнение (18) сводится к уравнению

$$
\hbar \omega_{e} \frac{\tilde{n}_{e}-\left\langle n_{e}\right\rangle}{\tau_{e}}-\hbar \omega_{f} \frac{\left\langle n_{f}\right\rangle}{\tau_{f}}+\hbar \Omega_{m} \frac{\tilde{n}_{m}-\left\langle n_{m}\right\rangle}{\tau_{m}}=0 .
$$

С другой стороны, имеет место закон сохранения для числа возбуждений

$$
\sum_{k} \frac{d\left\langle n_{k}\right\rangle}{d t}+\frac{d\left\langle S^{z}\right\rangle}{d t}=\sum_{k} \frac{\tilde{n}_{k}-\left\langle n_{k}\right\rangle}{\tau_{k}} .
$$

Легко видеть, что в стационарном режиме и при вышеуказанных условиях

$$
\frac{\tilde{n}_{e}-\left\langle n_{e}\right\rangle}{\tau_{e}}=\frac{\left\langle n_{f}\right\rangle}{\tau_{f}}
$$

Тогда из уравнения (19) следует, что

$$
\hbar\left(\omega_{f}-\omega_{e}\right) \frac{\tilde{n}_{e}-\left\langle n_{e}\right\rangle}{\tau_{e}}=\hbar \Omega_{m} \frac{\tilde{n}_{m}-\left\langle n_{m}\right\rangle}{\tau_{m}} .
$$

Рассматривая величину

$$
A=-\frac{\hbar \omega_{e}}{\tau_{e}} \tilde{n}_{e}
$$

как отрицательную работу, производимую внешним источником над подсистемой резонансных фононов для получения положительного тепла

$$
Q_{1}=\hbar \Omega_{m} \frac{\tilde{n}_{m}-\left\langle n_{m}\right\rangle}{\tau_{m}}
$$

от образца и возврашения со спонтанным излучением отрицательного тепла

$$
Q_{2}=-\hbar \omega_{e} \frac{\left\langle n_{e}\right\rangle}{\tau_{e}}-\hbar \omega_{f} \frac{\left\langle n_{f}\right\rangle}{\tau_{f}}
$$

окружающей среде, мы имеем обычное термодинамическое уравнение холодильной машины

$$
A=Q_{1}+Q_{2}
$$

где работа (возбуждение молекул на длинноволновом краю линии поглощения) затрачивается на передачу тепла от холодного тела (образец) к горячему (термостат), а рабочим телом является система резонансных фононов. Таким образом, внешнее лазерное 
излучение может охлаждать твердотельный образец через систему резонансных фононов. В неупорядоченных конденсированных системах подсистема примесей сама может служить охладителем для остального образца.

Построим уравнения для средних значений оператора числа вибронов в $m$-й моде и оператора неравновесной разности населенностей между электронными уровнями и, найдя стационарные решения этих уравнений, покажем возможность понижения температуры образца. Указанные операторы определим как $n_{j m}=B_{j m}^{+} B_{j m}$ и $p_{j}=2 S_{j}^{z}$ соответственно.

Сравнивая модельный гамильтониан $(14)$ с (1), видим, что операторам $M_{k}$ и $M_{k}^{+}$отвечают выражения

$$
\begin{aligned}
& M_{k}=g_{k} \sum_{j} e^{i \mathbf{k r}_{j}} S_{j}^{+}\left(1+\sum_{m}\left(\frac{h_{m k}}{g_{k}}\right)\left(B_{j m}+B_{j m}^{+}\right)\right), \\
& M_{k}^{+}=g_{k}^{*} \sum_{j} e^{-i \mathbf{k r}_{j}} S_{j}^{-}\left(1+\sum_{m}\left(\frac{h_{m k}}{g_{k}}\right)^{*}\left(B_{j m}+B_{j m}^{+}\right)\right) .
\end{aligned}
$$

Предполагая, что фотоны быстро (времена распространения фотонов в образце меньше всех характерных времен системы) уходят из образца, т.е. среднее число фотонов моды $k$, заданное внешним источником, совпадает со средним числом фотонов в системе, переходя к коллективным операторам излучателей и используя уравнение (13), запишем окончательные искомые уравнения для выбранных динамических величин (значок $\langle\cdot\rangle$ обозначает макроскопическое усреднение):

$$
\begin{aligned}
\frac{\left\langle d n_{m}\right\rangle}{d t}= & \frac{N}{2 \tau_{1 m}^{\mathrm{s}}}(\langle p\rangle+1)\left(\left\langle n_{m}\right\rangle+1\right)+\frac{N}{\tau_{m}^{\mathrm{s}}}\left[\langle p\rangle\left\langle n_{m}\right\rangle+\frac{1}{2}(\langle p\rangle+1)\right]- \\
& -\frac{N}{2 \tau_{1 m}^{\mathrm{as}}}(\langle p\rangle+1)\left\langle n_{m}\right\rangle-\frac{N}{\tau_{m}^{\mathrm{as}}}\left[\langle p\rangle\left\langle n_{m}\right\rangle+\frac{1}{2}(\langle p\rangle-1)\right]-\frac{1}{\tau_{m}^{\mathrm{v}}}\left(\left\langle n_{m}\right\rangle-n_{\mathrm{s}}\right), \\
\frac{d\langle p\rangle}{d t}= & -(\langle p\rangle+1)\left\{\sum_{m}\left[\frac{1}{\tau_{1 m}^{\mathrm{s}}}\left(\left\langle n_{m}\right\rangle+1\right)+\frac{1}{\tau_{1 m}^{\mathrm{as}}}\left\langle n_{m}\right\rangle+\frac{1}{\tau_{m}^{\mathrm{s}}}\right]+\frac{1}{\tau_{1}}\right\}- \\
& -2\langle p\rangle\left\{\sum_{m}\left[\frac{1}{\tau_{m}^{\mathrm{s}}}+\frac{1}{\tau_{m}^{\mathrm{as}}}\right]\left\langle n_{m}\right\rangle+\frac{1}{\tau_{p}}\right\}-\sum_{m} \frac{1}{\tau_{m}^{\mathrm{as}}}(\langle p\rangle-1),
\end{aligned}
$$

где $p$ - неравновесная разность населенностей между электронными уровнями, $n_{\mathrm{S}}-$ некое равновесное распределение фононов в образце, находящемся в термодинамическом равновесии с окружаюшей средой,

$$
\frac{1}{\tau_{1}}=\frac{2 \pi}{\hbar^{2}} \sum_{k}\left|g_{k}\right|^{2} \delta\left(\omega_{0}-\omega_{k}\right)
$$

- обратное время спонтанного излучения на прямом переходе,

$$
\frac{1}{\tau_{p}}=\frac{2 \pi}{\hbar^{2}} \sum_{k}\left\langle n_{k}\right\rangle\left|g_{k}\right|^{2} \delta\left(\omega_{0}-\omega_{k}\right)
$$


- обратное время стимулированного излучения на прямом переходе,

$$
\frac{1}{\tau_{1 m}^{\mathrm{s}}}=\frac{2 \pi}{\hbar^{2}} \sum_{k}\left|h_{k m}\right|^{2} \delta\left(\omega_{0}-\omega_{k}-\Omega_{m}\right)
$$

- обратное время спонтанного излучения на стоксовом прямом переходе при участии фонона моды $m$,

$$
\frac{1}{\tau_{1 m}^{\mathrm{as}}}=\frac{2 \pi}{\hbar^{2}} \sum_{k}\left|h_{k m}\right|^{2} \delta\left(\omega_{0}-\omega_{k}+\Omega_{m}\right)
$$

- обратное время спонтанного излучения на антистоксовом прямом переходе при участии фонона моды $m$,

$$
\frac{1}{\tau_{m}^{\mathrm{s}}}=\frac{2 \pi}{\hbar^{2}} \sum_{k}\left\langle n_{k}\right\rangle\left|h_{k m}\right|^{2} \delta\left(\omega_{0}-\omega_{k}-\Omega_{m}\right)
$$

- обратное время вьнужденного излучения на стоксовом прямом переходе при участии фонона моды $m$,

$$
\frac{1}{\tau_{m}^{\mathrm{as}}}=\frac{2 \pi}{\hbar^{2}} \sum_{k}\left\langle n_{k}\right\rangle\left|h_{k m}\right|^{2} \delta\left(\omega_{0}-\omega_{k}+\Omega_{m}\right)
$$

- обратное время вынужденного излучения на антистоксовом прямом переходе при участии фонона моды $m, 1 / \tau_{m}^{\mathrm{v}}$ - обратное время релаксации фононов моды $m$ к термодинамически равновесному состоянию.

Практические результаты могут быть получены в модели локальных или так называемых псевдолокализованных фононов, когда задача сводится фактически к четырехуровневой схеме. Псевдолокализованные фононы - это кванты комбинированного колебательного движения. Они образованы низкочастотными оптическими либрациями анизотропных примесных молекул, которые модулируют постоянную электрон-фотонного взаимодействия, и резонансными по отношению к ним акустическими фононами основного кристалла. Мы будем говорить о них как о резонансных фононах. Они характеризуются повышенной спектральной плотностью в узком спектральном диапазоне и проявляют себя как узкие пики в длинноволновом крыле линии поглощения.

Рассмотрим возбуждения на стоксовом переходе. В этом случае все числа фотонов, за исключением фотонов с частотой $\omega_{0}-\Omega_{0}$, должны быть положены равными нулю. $\mathrm{B}$ приближении низких температур, $\left\langle n_{\mathrm{s}}\right\rangle \ll 1$, можно показать, что антистоксовы процессы проявляют себя лишь во втором порядке малости по среднему числу фононов всевозможных мод, определяемом взаимодействием с термостатом. В этих приближениях и когда поле источника не слишком мало, $\left\langle n_{k}\right\rangle \gg 1$ и $\tau_{\mathrm{s}} \ll \tau_{1}^{\mathrm{s}}\left(\tau_{\mathrm{s}}^{-1}-\right.$ обратное время вынуж денного излучения на стоксовом прямом переходе при участии фононов всевозможных мод, $\left(\tau_{1}^{\mathrm{S}}\right)^{-1}$ - обратное время спонтанного излучения на стоксовом прямом переходе при участии фононов всевозможных мод), мы можем переписать систему уравнений (29), (30) в виде

$$
\begin{aligned}
& \frac{d\langle n\rangle}{d t}=\frac{N}{\tau_{\mathrm{s}}}\left[\langle p\rangle\langle n\rangle+\frac{1}{2}(\langle p\rangle+1)\right]-\frac{1}{\tau_{\mathrm{v}}}\left(\langle n\rangle-\left\langle n_{\mathrm{s}}\right\rangle\right), \\
& \frac{d\langle p\rangle}{d t}=-\frac{1}{\tau_{1}}(\langle p\rangle+1)-2 \frac{M}{\tau_{\mathrm{s}}}\left(\langle p\rangle\langle n\rangle+\frac{\langle p\rangle+1}{2}\right),
\end{aligned}
$$


где $\left\langle n_{\mathrm{S}}\right\rangle$ - среднее число резонансных фононов, $\tau_{\mathrm{v}}$ - время их термализации, $M$ - количество фононных состояний, участвующих в охлаждении.

Стационарное решение системы $(37),(38)$

$$
\begin{aligned}
\langle n\rangle= & \frac{\tau_{\mathrm{S}} \tau_{\mathrm{v}}}{M}\left\{-\left(\frac{1}{\tau_{1} \tau_{\mathrm{v}}}+\frac{1}{\tau_{\mathrm{s}} \tau_{1}}+\frac{M}{\tau_{\mathrm{s}} \tau_{\mathrm{v}}}\right)+\frac{2 M}{\tau_{\mathrm{s}} \tau_{\mathrm{v}}}\left\langle n_{\mathrm{s}}\right\rangle+\right. \\
& \left.+\left[\left(\frac{1}{\tau_{1} \tau_{\mathrm{v}}}+\frac{1}{\tau_{\mathrm{s}} \tau_{1}}+\frac{M}{\tau_{\mathrm{s}} \tau_{\mathrm{v}}}-\frac{2 M}{\tau_{\mathrm{s}} \tau_{\mathrm{v}}}\left\langle n_{\mathrm{S}}\right\rangle\right)^{2}+\frac{8 M}{\tau_{\mathrm{s}} \tau_{\mathrm{v}}^{2}}\left(\frac{M}{\tau_{\mathrm{S}}}+\frac{1}{\tau_{1}}\right)\left\langle n_{\mathrm{s}}\right\rangle\right]^{\frac{1}{2}}\right\}
\end{aligned}
$$

в приближении низких температур имеет вид

$$
\langle n\rangle=\frac{\tau_{\mathrm{v}}^{-1}\left(M \tau_{\mathrm{s}}^{-1}+\tau_{1}^{-1}\right)\left\langle n_{\mathrm{s}}\right\rangle}{\tau_{\mathrm{v}}^{-1}\left(M \tau_{\mathrm{s}}^{-1}+\tau_{1}^{-1}\right)+N\left(\tau_{1} \tau_{\mathrm{S}}\right)^{-1}} .
$$

Отсюда при достаточно больших временах $N \tau_{\mathrm{v}} \ll M \tau_{1}$ имеем

$$
\langle n\rangle=n_{\mathrm{s}} \frac{\tau_{1}}{\tau_{\mathrm{v}}} \frac{M}{N}
$$

и для эффективной температуры -

$$
T=T_{\mathrm{s}}\left(1+\frac{k_{\mathrm{B}} T_{\mathrm{S}}}{\hbar \Omega_{0}} \ln \left(\frac{\tau_{\mathrm{v}}}{\tau_{1}} \frac{N}{M}\right)\right)^{-1},
$$

где $T_{\mathrm{S}}$ - температура образца.

В высокотемпературном приближении, $\left\langle n_{\mathrm{S}}\right\rangle \gg 1$, стационарное решение уравнений (29), (30) для $\langle n\rangle$ имеет вид

$\langle n\rangle=\left\langle n_{\mathrm{S}}\right\rangle-$

$$
-\left(\frac{N}{M}\right) \frac{\left(\tau_{1}\right)^{-1}\left(2\left(\tau_{\mathrm{S}}\right)^{-1}+\left(\tau_{1}^{\mathrm{S}}\right)^{-1}\right)-2 M\left(\tau_{1}^{\mathrm{as}}\right)^{-1}\left(\left(\tau_{1}\right)^{-1}+\left(\tau_{\mathrm{S}}\right)^{-1}+\left(\tau_{1}^{\mathrm{s}}\right)^{-1}\right)}{\tau_{\mathrm{S}}\left(\tau_{\mathrm{v}}\right)^{-1}\left(2\left(\tau_{\mathrm{S}}\right)^{-1}+\left(\tau_{1}^{\mathrm{S}}\right)^{-1}+\left(\tau_{1}^{\mathrm{as}}\right)^{-1}\right)^{2}}
$$

и при $\tau_{\mathrm{s}} \ll \tau_{1}^{\mathrm{s}}, \tau_{1}^{\mathrm{as}} ; \tau_{1} \ll \tau_{1}^{\mathrm{as}} / M$ сводится к

$$
\begin{gathered}
\langle n\rangle=n_{0}-\frac{\tau_{\mathrm{v}}}{\tau_{1}} \frac{N}{M}, \\
T=T_{\mathrm{s}}\left(1-\frac{\hbar \Omega_{0}}{k_{\mathrm{B}} T_{\mathrm{s}}} \frac{\tau_{\mathrm{v}} N}{\tau_{1} M}\right) .
\end{gathered}
$$

Оценим величину охлаждения для флюридного стекла, легированного ионами иттербия с концентрацией в один весовой процент [8]. Полагая $N=10^{16} \mathrm{~cm}^{-3}, \tau_{\mathrm{v}} \approx \tau_{1}$ и порядка $10^{-5} \mathrm{c}, M=2.5 \times 10^{16} \mathrm{~cm}^{-3}[15], \Omega_{0}=61$ ГГц, получаем, что возможно охлаждение указанного образца от комнатной температуры $(298 \mathrm{~K})$ до $269 \mathrm{~K}$, что удовлетворительно согласуется с результатами эксперимента [8].

Благодарности. Авторы считают приятным долгом поблагодарить Е. К. Башкирова за идею использовать метод исключения бозонных переменных и полезные обсуждения. Данная работа поддержана грантом РФФИ № 99-02-18495а, грантами МНТЦ РФ “Физика квантовых и волновых процессов: Фундаментальная спектроскопия и Лазерная физика" и грантом Федеральной программы "Университеты России - Фундаментальные исследования" (№ 990214). 


\section{Список литературы}

[1] С.Н. Андрианов, В.В. Самарцев. Оптическое сверхизлучение и лазерное охлаждение в твердых телах. Казань: Изд. КГУ, 1998.

[2] В. Г. Миногин, В. С. Летохов. Давление лазерного излучения на атомы. М.: Наука, 1986.

[3] А. П. Казанцев, Г. И. Сурданович, В. П. Яжовлев. Механическое действие света на атомы. М.: Наука, 1991.

[4] P. Pringsheim. Z. Phys. 1929. V. 57. P. 739.

[5] A. Kastler. J. Phys. Radium. 1950. V. 11. P. 250.

[6] R.I. Epstein, M.I. Buchwald, B. C. Edwards, T.R. Gosnell, C.E. Mungan. Nature. 1995. V. 377. P. 500.

[7] C. E. Mungan, T.R. Gosnell. Advances in Atomic, Molecular and Optical Physics (review). 1999. V. 40. P. 161.

[8] X. Luo, M. D. Eisaman, T. R. Gosnell. Optics Lett. 1998. V. 23. № 8. P. 639.

[9] R. Epstein, S. Bradley, C. Edwards, M. Buchwald, R. Gosnell. Fluerescent refrigeration. Patent USA, № 5.447.032 (Date: 05.09.95).

[10] S. N. Andrianov, V. V. Samartsev. Laser Phys. 1996. V. 6. № 5. P. 949.

[11] S. N. Andrianov, V. V. Samartsev. Laser Phys. 1996. V. 6. № 4. P. 759.

[12] A. A. Kalachev, S. B. Karamyshev, V. V. Samartsev. Laser Phys. 1996. V. 6. № 1. P. 27.

[13] A. A. Kalachev, V. V. Samartsev. Laser Phys. 1997. V. 7. № 2. P. 1.

[14] S. N. Andrianov, V. V. Samartsev. Laser Phys. 1998. V. 8. № 1. P. 14.

[15] S. N. Andrianov, V. V. Samartsev. Laser Phys. 1999. V. 9. № 5. P. 1021.

[16] В. М. Файн, Я.И. Ханин. Квантовая радиофизика: фотоны и нелинейные среды. М.: Сов. радио, 1972

[17] N. N. Bogolubov. Kinetic equations for the electron-phonon system. Preprint E17-11822. Dubna: JINR, 1978

[18] Н. Н. Боголюбов, Н. Н. Боголюбов (мл.). ЭЧАЯ. 1980. Т. 11. С. 245.

[19] Н. Н. Боголюбов, Н. Н. Боголюбов (мл.). Введение в квантовую статистическую механику. М.: Наука, 1984.

[20] Н. Н. Боголюбов (мл.), А. С. Шумовский. Сверхизлучение. Дубна: ОИЯИ, 1987.

[21] E. K. Bashkirov, S. V. Petrushkin. Quantum correlation functions in the problem of collective spontaneous emission. In: Proc. of the Workshop QFTHEP, 1997. Ed. B. B. Levtchenko. M. INP MSU, 1998. P. 466.

[22] Y.R. Shen. Phys. Rev. 1967. V. 155. P. 921.

[23] D. F. Walls. J. Phys. A. 1971. V. 4. P. 813.

[24] K. J. McNeil, D. F. Walls. J. Phys. A. 1975. V. 8. P. 104.

[25] K. J. McNeil, D. F. Walls. J. Phys. A. 1975. V. 8. P. 111.

[26] А.И. Ахиезер, С. В. Пелетминский. Методы статистической физики. М.: Наука, 1977.

Поступила в редакцию 5.V.2000 г., после доработки 27.VI.2000 г. 